\title{
Factors Prognostic of Season-Associated Sudden Sensorineural Hearing Loss: A Retrospective Observational Study
}

\author{
In Yong Ryu, Sang Hyun Park, Eun Bin Park, Ho Joong Kim, \\ Sang Hoon Kim, and Seung Geun Yeo \\ Department of Otorhinolaryngology, Graduate School, Kyung Hee University, Seoul, Korea
}

\author{
Received June 10,2016 \\ Revised September 12, 2016 \\ Accepted September 26, 2016
Address for correspondence
Seung Geun Yeo, MD, PhD
Department of Otorhinolaryngology,
Graduate School,
Kyung Hee University,
23 Kyungheedae-ro,
Dongdaemun-gu, Seoul 02447,
Korea
Tel $+82-2-958-8474$
Fax $+82-2-958-8470$ \\ E-mail yeo2park@gmail.com
}

\begin{abstract}
Background and Objectives: This study examined whether the prognosis of sudden deafness was dependent on the time of onset and evaluated the factors affecting prognosis during each period. Subjects and Methods: Patients who developed sudden hearing loss from January 2005 to December 2015 were evaluated retrospectively. Meteorological data were obtained from the official website of the Korea Meteorological Administration. Factors prognostic of hearing recovery rate were analyzed. Results: The hearing recovery rate of the 318 patients who developed sudden deafness did not differ significantly by month Mean temperature and daily temperature range at onset of sudden deafness were not as sociated with recovery rate. Conclusions: The recovery rate in patients with sudden deafness did not differ markedly by season.

J Audiol Otol 2017;21(1):44-48

KEY WORDS: Sudden sensorineural hearing loss · Season · Prognostic factor.
\end{abstract}

\section{Introduction}

Sudden deafness is a type of hearing loss without obvious cause occurring over a relatively short period of time. It is diagnosed in subjects who experience a continuous reduction in hearing loss at $\geq 30 \mathrm{~dB}$ at more than three consecutive frequencies for 12 hours to 3 days. Unless promptly treated, it can cause severe disorders, such as permanent hearing impairment, tinnitus, and psychiatric sequelae. This disease has been reported to affect approximately 5 to 20 people per 100,000 per year, with a spontaneous recovery rate of $32 \%$ to $65 \%[1-3]$.

Relatively little is known about the factors associated with hearing recovery following sudden deafness. Moreover, the pathogenesis of this condition remains to be established. Etiologic factors include viruses, circulatory problems in the anterior inferior cerebellar artery, acoustic neuromas, and peri-

This is an Open Access article distributed under the terms of the Creative Commons Attribution Non-Commercial License (http://creativecommons.org/licenses/by-nc/4.0/) which permits unrestricted non-commercial use, distribution, and reproduction in any medium, provided the original work is properly cited. lymph fistulae, along with environmental, dietary, and seasonal factors [4-6]. Seasonal climatic changes, which have long been known to affect human health [7], can affect both the development of and recovery from sudden deafness [8-10]. Thus, the pathophysiology of sudden deafness may depend on the time of onset. This study therefore evaluated whether the prognosis of sudden deafness was dependent on the season of onset, and assessed the factors affecting prognosis during each season.

\section{Subjects and Methods}

This study retrospectively evaluated patients living in Seoul, South Korea, who visited an otorhinolaryngological clinic and received inpatient treatment for sudden deafness from January 2005 to December 2015. The diagnosis of sudden deafness was based on history taking and pure tone audiometry (hearing loss at $\geq 30 \mathrm{~dB}$ at more than three consecutive frequencies). Patients with an uncertain date of onset and those who began treatment more than 15 days after onset were excluded. 
Patients who had not previously received intratympanic steroid injections and those who refused additional treatment were hospitalized for 6-8 days, fed with a low salt diet, and treated with systemic oral steroids, consisting of $80 \mathrm{mg} /$ day methylprednisolone (Methylon tab $4 \mathrm{mg}$ ) on days 1 to 4,60 $\mathrm{mg}$ /day on days 5 and 6, $40 \mathrm{mg}$ /day on days 7 and $8,20 \mathrm{mg} /$ day on days 9 and 10 , and $10 \mathrm{mg} /$ day on days 11 to 14 . During the period of steroid administration, patients were prescribed almitrine bismesylate and buflomedil pyridoxal phosphate to improve blood circulation.

Other patients were treated with both oral steroids and intratympanic steroid injections. These patients received four injections at outpatient clinics at 3-4 day intervals; prior to each injection, change in hearing was assessed by pure tone audiometry. With the patient in a supine position and the ear facing up, 0.3-0.4 cc dexamethasone (Dexamethasone ${ }^{\circledR}$; Jeil pharmaceutical, Daegu, Korea, $5 \mathrm{mg} / \mathrm{mL}$ ) was injected under microscopic guidance into the anterior inferior quadrant area of the ear drum on the affected side using a 25-gauge spinal needle connected to a $1 \mathrm{cc}$ syringe. Patients were instructed to remain supine for 20 minutes and not to swallow saliva or talk during this period.

The level of recovery was evaluated by pure tone audiometry as described [9]. Complete recovery (CR) was defined as a final pure tone audiometry result $<25 \mathrm{~dB}$; and partial recovery (PR) as a $>15 \mathrm{~dB}$ improvement, but final hearing of 25-45 dB. Slight improvement (SI) was defined as a $>15 \mathrm{~dB}$ improvement, but final hearing $>45 \mathrm{~dB}$; and no improvement (NI) as hearing improvement $<15 \mathrm{~dB}$ and final hearing $>75$ dB. Patients who achieved CR, PR, and SI were defined as those who recovered, whereas patients with NI were defined as unrecovered. The mean follow-up period after onset of sudden deafness was three months.

Patients with seasonal changes were defined as those who developed sudden deafness from the start of spring to the start of summer and from the start of autumn to the start of winter. Patients without seasonal changes were defined as those who developed sudden deafness from the start of summer to the start of autumn and from the start of winter to the start of spring.

Factors analyzed as possibly associated with hearing recovery rate after treatment included the presence or absence of associated symptoms, such as diabetes mellitus, hypertension, otitis media, upper respiratory tract infection, tinnitus, dizziness, and aural fullness. Other factors included white blood cell and neutrophil counts at the time of hospital admission and daily temperature range and mean daily temperature for 15 days, starting 1 week before the onset of sudden deafness to 1 week after onset, based on data recorded on the official website of the Korea Meteorological Administration (www.kma.go.kr).

All statistical analyses were performed using SPSS version 18.0 (SPSS Inc., Chicago, IL, USA). Groups were compared by ANOVA, the chi-square test, and Fisher's exact test, as appropriate. A $p$-value $<0.05$ was considered statistically significant.

\section{Results}

The study population consisted of 318 patients, 191 (60.1\%)

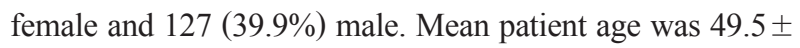
15.7 years. According to Siegel's criteria, 115 (36.2\%) patients experienced CR, 44 (13.8\%) experienced PR, 51 (16.0\%) experienced slight recovery, and $108(34.0 \%)$ showed no evidence of recovery (Table 1).

Assessment of the relationship between intra-annual distribution of onset of sudden deafness and hearing recovery showed no statistically significant differences by month (Fig. 1). There are several items related with SSNHL. We analyzed the time from the onset of SSNHL to first visit to ENT clinics,

Table 1. Baseline demographic and clinical characteristics of patients with sudden hearing loss $(n=318)$

\begin{tabular}{lc}
\hline \multicolumn{1}{c}{ Variables } & Number (\%) \\
\hline Gender & $191(60.1)$ \\
Female & $127(39.9)$ \\
Male & $49.5 \pm 15.7$ \\
Age (years, mean \pm SD) & \\
Past medical history & $37(11.6)$ \\
DM & $89(28.0)$ \\
HTN & $14(4.4)$ \\
COM Hx & $12(3.8)$ \\
URI & \\
Prevalence of associated symptoms & $246(77.8)$ \\
Tinnitus & $83(26.2)$ \\
Dizziness & $151(47.9)$ \\
Ear fullness & \\
Laboratory data & $8.45 \pm 3.345$ \\
WBC (/ $\mu L$, mean $\pm S D)$ & $66.9 \pm 14.6$ \\
Neutrophil (\%, mean $\pm S D)$ & $4.13 \pm 3.43$ \\
Time from onset of SSNHL to the first visit & \\
to ENT clinic (days, mean $\pm S D)$ & $115(36.2)$ \\
Recovery & $44(13.8)$ \\
Complete & $51(16.0)$ \\
Partial & $108(34.0)$ \\
Slight & \\
No improvement & \\
\hline SSNH: sudden sensory neur & \\
\hline
\end{tabular}

SSNHL: sudden sensory neural hearing loss, DM: diabetes mellitus, HTN: hypertension, COM Hx: chronic otitis media history, URI: upper respiratory infection, WBC: urine white blood cell 


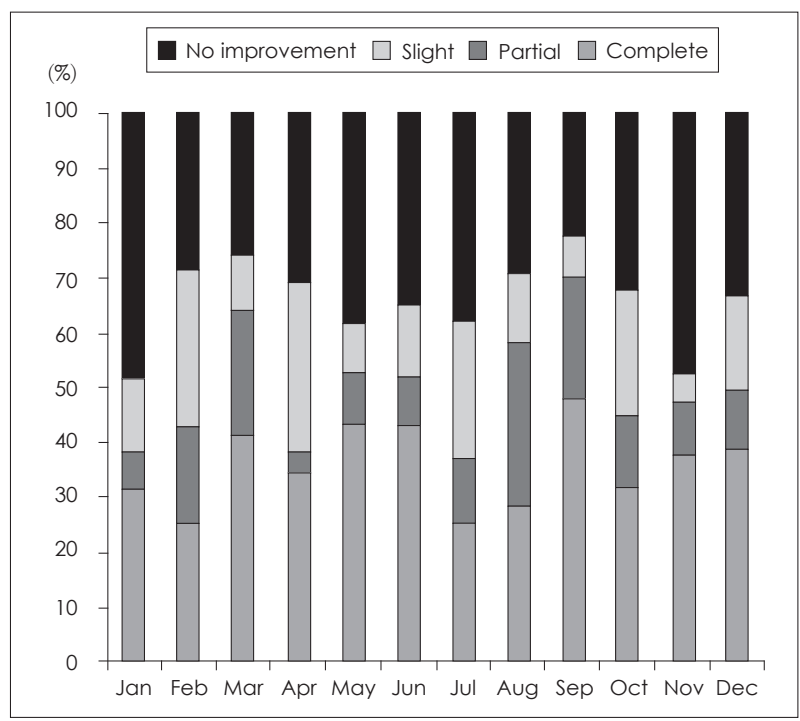

Fig. 1. Monthly recovery rate of SSNHL. SSNHL: sudden sensory neural hearing loss.
WBC, Neutrophil, DM, HTN, tinnitus, dizziness and ear fullness. Only the time from onset of SSNHL to first visit to ENT clinic and dizziness were associated with SSNHL and statistically significant (Table 2). However, meteorological and laboratory parameters were not correlated with recovery rate (Table 3). Moreover, the mean temperature and daily temperature before and after the date of onset of sudden deafness did not correlate with recovery rate (Table 4).

\section{Discussion}

Sudden deafness is characterized by symptoms of sensorineural deafness without an obvious cause, occurring over hours to days and frequently accompanied by symptoms such as tinnitus and dizziness. All patients in this study were from Seoul, the capital of South Korea located at 37.33 degrees north latitude. The mean annual temperature in Seoul ranges

Table 2. Relationships of clinical characteristics and recovery rates in patients with SSNHL

\begin{tabular}{lccccc}
\hline & Complete & Partial & Slight & No improvement & $p$ value \\
\hline Time from onset of SSNHL to first visit to ENT clinic & $3.75 \pm 2.87$ & $5.38 \pm 3.19$ & $2.80 \pm 2.50$ & $4.61 \pm 4.14$ & $0.001^{*}$ \\
WBC $(/ \mu \mathrm{L})$ & $8.26 \pm 3.23$ & $8.13 \pm 3.66$ & $8.58 \pm 3.54$ & $8.69 \pm 3.30$ & 0.711 \\
Neutrophil (\%) & $66.42 \pm 14.12$ & $63.96 \pm 17.83$ & $64.77 \pm 16.45$ & $68.97 \pm 13.97$ & 0.187 \\
DM (\%) & 8.7 & 24.4 & 13.7 & 10.2 & 0.053 \\
HTN (\%) & 21.7 & 40.0 & 37.3 & 26.9 & 0.062 \\
Tinnitus (\%) & 81.6 & 68.9 & 74.0 & 80.6 & 0.288 \\
Dizziness (\%) & 11.3 & 31.1 & 48.0 & 31.5 & $0.001^{*}$ \\
Ear fullness (\%) & 51.8 & 53.3 & 46.9 & 43.5 & 0.581 \\
\hline
\end{tabular}

*p<0.05. SSNHL: sudden sensory neural hearing loss, DM: diabetes mellitus, HTN: hypertension

Table 3. Relationships of clinical characteristics and recovery rates in patients with SSNHL according to season

\begin{tabular}{|c|c|c|c|c|c|}
\hline & Complete & Partial & Slight & No improvement & $p$ value \\
\hline \multicolumn{6}{|l|}{ Spring } \\
\hline Mean temperature at onset & $6.43 \pm 3.64$ & $4.82 \pm 4.23$ & $7.98 \pm 6.71$ & $7.25 \pm 4.47$ & 0.410 \\
\hline Daily temperature range at onset & $8.16 \pm 2.90$ & $8.02 \pm 2.65$ & $9.12 \pm 3.19$ & $8.87 \pm 2.32$ & 0.637 \\
\hline $\mathrm{WBC}(/ \mu \mathrm{L})$ & $7.742 \pm 2.923$ & $7.654 \pm 2.743$ & $9.515 \pm 4.391$ & $8.542 \pm 3.983$ & 0.346 \\
\hline Neutrophil (\%) & $66.03 \pm 12.67$ & $62.78 \pm 13.70$ & $68.28 \pm 15.33$ & $69.29 \pm 16.91$ & 0.595 \\
\hline \multicolumn{6}{|l|}{ Summer } \\
\hline Mean temperature at onset & $20.18 \pm 4.19$ & $22.05 \pm 2.45$ & $20.14 \pm 8.03$ & $19.45 \pm 5.74$ & 0.835 \\
\hline Daily temperature range at onset & $7.30 \pm 3.21$ & $8.13 \pm 3.35$ & $6.53 \pm 4.07$ & $7.68 \pm 2.85$ & 0.785 \\
\hline $\mathrm{WBC}(/ \mu \mathrm{L})$ & $8.885 \pm 3.273$ & $8.464 \pm 5.409$ & $7.123 \pm 1.328$ & $8.077 \pm 2.740$ & 0.382 \\
\hline Neutrophil (\%) & $67.93 \pm 12.74$ & $69.68 \pm 15.14$ & $59.98 \pm 18.87$ & $68.23 \pm 14.46$ & 0.317 \\
\hline \multicolumn{6}{|l|}{ Fall } \\
\hline Mean temperature at onset & $19.06 \pm 5.09$ & $20.85 \pm 5.14$ & $19.54 \pm 5.13$ & $18.06 \pm 7.05$ & 0.594 \\
\hline Daily temperature range at onset & $7.70 \pm 2.18$ & $7.82 \pm 2.89$ & $8.61 \pm 3.16$ & $7.99 \pm 2.34$ & 0.810 \\
\hline $\mathrm{WBC}(/ \mu \mathrm{L})$ & $8.042 \pm 2.802$ & $9.207 \pm 3.468$ & $9.089 \pm 4.195$ & $8.927 \pm 2.658$ & 0.557 \\
\hline Neutrophil (\%) & $66.80 \pm 11.26$ & $62.96 \pm 15.71$ & $66.23 \pm 15.89$ & $71.40 \pm 13.71$ & 0.261 \\
\hline \multicolumn{6}{|l|}{ Winter } \\
\hline Mean temperature at onset & $2.34 \pm 5.71$ & $3.10 \pm 4.84$ & $0.51 \pm 4.84$ & $2.78 \pm 5.34$ & 0.789 \\
\hline Daily temperature range at onset & $7.55 \pm 2.13$ & $5.60 \pm 2.16$ & $7.70 \pm 1.64$ & $7.33 \pm 1.73$ & 0.218 \\
\hline $\mathrm{WBC}(/ \mu \mathrm{L})$ & $8.572 \pm 3.816$ & $6.710 \pm 2.450$ & $7.820 \pm 1.407$ & $9.531 \pm 3.695$ & 0.247 \\
\hline Neutrophil (\%) & $67.12 \pm 15.31$ & $72.07 \pm 18.21$ & $62.40 \pm 15.34$ & $68.44 \pm 14.49$ & 0.480 \\
\hline
\end{tabular}

SSNHL: sudden sensory neural hearing loss, WBC: urine white blood cell 
Ryu IY, et al.

Table 4. Relationships of daily temperature range and mean temperature in patients with SSNHL according to season

\begin{tabular}{|c|c|c|c|c|c|c|}
\hline Variables & Day & Complete & Partial & Slight & No improvement & $p$ value \\
\hline Mean temperature around onset of & -7 & $8.25 \pm 2.80$ & $7.94 \pm 2.55$ & $8.23 \pm 2.63$ & $8.08 \pm 2.60$ & 0.935 \\
\hline \multirow[t]{14}{*}{ sudden deafness } & -6 & $7.97 \pm 2.41$ & $8.40 \pm 2.88$ & $8.43 \pm 2.94$ & $8.59 \pm 2.86$ & 0.467 \\
\hline & -5 & $8.12 \pm 2.35$ & $8.10 \pm 2.31$ & $7.48 \pm 2.25$ & $7.36 \pm 2.60$ & 0.135 \\
\hline & -4 & $8.21 \pm 2.68$ & $8.73 \pm 2.66$ & $7.97 \pm 2.45$ & $7.94 \pm 2.92$ & 0.536 \\
\hline & -3 & $8.21 \pm 2.76$ & $7.73 \pm 2.06$ & $7.80 \pm 2.46$ & $7.77 \pm 2.63$ & 0.630 \\
\hline & -2 & $7.91 \pm 2.60$ & $7.47 \pm 2.94$ & $7.88 \pm 2.55$ & $7.88 \pm 2.55$ & 0.325 \\
\hline & -1 & $7.98 \pm 2.81$ & $6.99 \pm 2.80$ & $7.86 \pm 2.93$ & $8.10 \pm 2.49$ & 0.251 \\
\hline & Onset & $7.67 \pm 2.62$ & $7.57 \pm 2.78$ & $8.11 \pm 3.27$ & $7.96 \pm 2.38$ & 0.736 \\
\hline & +1 & $7.92 \pm 2.66$ & $7.69 \pm 2.44$ & $8.19 \pm 2.52$ & $8.03 \pm 2.52$ & 0.728 \\
\hline & +2 & $7.74 \pm 2.75$ & $8.24 \pm 2.36$ & $8.52 \pm 2.92$ & $8.24 \pm 2.60$ & 0.392 \\
\hline & +3 & $7.78 \pm 2.66$ & $7.43 \pm 2.25$ & $8.70 \pm 2.54$ & $8.02 \pm 2.74$ & 0.185 \\
\hline & +4 & $8.26 \pm 2.69$ & $8.19 \pm 2.31$ & $8.89 \pm 3.41$ & $8.39 \pm 2.59$ & 0.634 \\
\hline & +5 & $8.28 \pm 2.65$ & $7.53 \pm 2.48$ & $9.03 \pm 3.02$ & $7.99 \pm 2.52$ & 0.092 \\
\hline & +6 & $8.14 \pm 2.30$ & $8.25 \pm 2.09$ & $7.63 \pm 2.69$ & $8.42 \pm 2.63$ & 0.422 \\
\hline & +7 & $8.35 \pm 2.63$ & $7.99 \pm 3.23$ & $7.39 \pm 2.29$ & $8.29 \pm 2.68$ & 0.264 \\
\hline Daily temperature range around onset & -7 & $12.28 \pm 10.10$ & $12.63 \pm 10.92$ & $12.23 \pm 11.61$ & $11.71 \pm 9.70$ & 0.971 \\
\hline \multirow[t]{14}{*}{ of sudden deafness } & -6 & $12.56 \pm 10.00$ & $13.42 \pm 10.55$ & $12.29 \pm 12.50$ & $12.16 \pm 9.73$ & 0.948 \\
\hline & -5 & $12.50 \pm 9.80$ & $13.41 \pm 10.69$ & $12.00 \pm 11.17$ & $12.14 \pm 10.01$ & 0.932 \\
\hline & -4 & $12.74 \pm 10.46$ & $13.47 \pm 11.33$ & $12.11 \pm 10.82$ & $11.92 \pm 10.02$ & 0.888 \\
\hline & -3 & $12.67 \pm 9.97$ & $12.89 \pm 11.18$ & $12.01 \pm 10.61$ & $11.40 \pm 9.92$ & 0.825 \\
\hline & -2 & $12.31 \pm 9.69$ & $12.75 \pm 10.83$ & $12.47 \pm 10.12$ & $12.05 \pm 10.22$ & 0.988 \\
\hline & -1 & $12.59 \pm 9.52$ & $12.92 \pm 10.60$ & $12.80 \pm 10.17$ & $12.26 \pm 9.65$ & 0.985 \\
\hline & Onset & $12.31 \pm 9.10$ & $13.22 \pm 9.63$ & $12.60 \pm 9.80$ & $12.34 \pm 9.07$ & 0.967 \\
\hline & +1 & $12.56 \pm 9.35$ & $12.25 \pm 10.97$ & $12.95 \pm 9.41$ & $12.34 \pm 9.15$ & 0.987 \\
\hline & +2 & $12.59 \pm 9.28$ & $12.09 \pm 11.66$ & $12.94 \pm 9.03$ & $12.45 \pm 9.52$ & 0.986 \\
\hline & +3 & $12.77 \pm 8.78$ & $11.93 \pm 11.71$ & $13.71 \pm 9.06$ & $12.25 \pm 9.80$ & 0.843 \\
\hline & +4 & $12.84 \pm 9.23$ & $12.28 \pm 11.18$ & $13.68 \pm 9.67$ & $12.46 \pm 10.05$ & 0.917 \\
\hline & +5 & $12.86 \pm 9.68$ & $12.17 \pm 10.55$ & $13.34 \pm 10.13$ & $12.45 \pm 10.05$ & 0.954 \\
\hline & +6 & $12.85 \pm 9.90$ & $12.77 \pm 10.29$ & $13.33 \pm 10.07$ & $12.15 \pm 10.40$ & 0.935 \\
\hline & +7 & $12.79 \pm 9.86$ & $12.55 \pm 9.72$ & $12.88 \pm 10.26$ & $12.01 \pm 10.06$ & 0.952 \\
\hline
\end{tabular}

SSNHL: sudden sensory neural hearing loss

from -6 to $-3^{\circ} \mathrm{C}$ during the coldest month (January) to 23 to $27^{\circ} \mathrm{C}$ during the hottest month (August). December, January, and February were defined as winter; March, April, and May as spring; June, July, and August as summer; and September, October, and November as autumn. This study found that recovery rates did not differ significantly among patients with onset during these four seasons. In contrast, a previous study reported that the hearing recovery rate was higher in patients with onset of sudden deafness during spring than during other seasons [11].

To reflect seasonal characteristics more in this study, patients were assorted with reference to 24 solar terms. A solar term is a calendar designed to reflect seasonal changes during the Zhou period in China and characterized by the sun's position along the ecliptic. The longest period of daylight was defined as the summer solstice, and the longest period of night as the winter solstice. The 24 solar terms were formed by evenly placing the other terms, with 6 solar terms each in spring, summer, autumn, and winter.

Factors previously associated with prognosis following sudden deafness include age, dizziness, tinnitus, and time from onset to treatment [11-15]. In the present study, when the patients were divided according to the degree of recovery from sudden deafness, the co-occurrence of dizziness and time from onset of sudden deafness to the first visit to the ENT clinic differed significantly.

Infection, includes virus, has been reported to be one of the etiological factors in sudden deafness [4-6]. However, despite of several investigations that are on the process, there was no evidence that a pathogen can directly cause sudden deafness $[16,17]$. Our study attempted to assess the impacts of infection on deafness by determining whether white blood 
cell counts and percentage of neutrophils differed by recovery rates. Although we were unable to detect any correlations, studies are needed to determine the etiological factors of sudden deafness. One limitation of this study was that history of upper respiratory tract infections in these patients was not analyzed.

Tinnitus has been reported not to be associated with prognosis [14] and to be associated with both an unfavorable $[13,15]$ and a favorable $[18,19]$ prognosis. In this study, tinnitus was not associated with recovery rate.

Many studies have assessed the relationship between climate and sudden deafness [20-22], but fewer have analyzed climatic factors closely related to sudden deafness. Changes in temperature may affect disease results after the onset of sudden deafness, the daily temperature range and average daily temperature from 7 days before to 7 days after the onset of sudden deafness were analyzed. But there was no statistically significant difference. These results confirm findings, showing that climate on the day of onset is unrelated to sudden deafness [20]. The etiologic association between daily temperature range and recovery from sudden hearing loss remains unclear.

In conclusion, the recovery rate in patients with sudden deafness did not differ significantly by month of inset. Mean temperature and daily temperature range around the onset of sudden deafness did not correlate with recovery rate.

\section{Acknowledgments}

This work was supported by the National Research Foundation of Korea (NRF) grant funded by the Korea government (MSIP) (No. 2011-0030072).

\section{Conflicts of interest}

The authors have no financial conflicts of interest.

\section{REFERENCES}

1) Wilson WR. Why treat sudden hearing loss. Am J Otol 1984;5:481-3.

2) Fetterman BL, Saunders JE, Luxford WM. Prognosis and treatment of sudden sensorineural hearing loss. Am J Otol 1996;17:529-36.

3) Mattox DE, Simmons FB. Natural history of sudden sensorineural hearing loss. Ann Otol Rhinol Laryngol 1977;86:463-80.

4) Huy PT, Sauvaget E. Idiopathic sudden sensorineural hearing loss is not an otologic emergency. Otol Neurotol 2005;26:896-902.

5) Nakashima T, Tanabe T, Yanagita N, Wakai K, Ohno Y. Risk fac- tors for sudden deafness: a case-control study. Auris Nasus Larynx 1997;24:265-70.

6) Nakamura M, Aoki N, Nakashima T, Hoshino T, Yokoyama T, Morioka S, et al. Smoking, alcohol, sleep and risk of idiopathic sudden deafness: a case-control study using pooled controls. J Epidemiol 2001;11:81-6.

7) Shutty MS Jr, Cundiff G, DeGood DE. Pain complaint and the weather: weather sensitivity and symptom complaints in chronic pain patients. Pain 1992;49:199-204.

8) Mees K, Ruhenstroth-Bauer G, Sandhagen R, Baumer H, Filipiak B. [Idiopathic hearing loss--dependent on the weather?]. Laryngol Rhinol Otol (Stuttg) 1987;66:246-8.

9) Danielides V, Nousia CS, Bartzokas A, Lolis CJ, Kateri M, Skevas A. Weather conditions and sudden sensorineural hearing loss. BMC Ear Nose Throat Disord 2002;2:2.

10) Preyer S. [Effect of weather on the incidence of sudden deafness]. Laryngorhinootologie 1996;75:443-6.

11) Narozny W, Kuczkowski J, Kot J, Stankiewicz C, Sicko Z, Mikaszewski B. Prognostic factors in sudden sensorineural hearing loss: our experience and a review of the literature. Ann Otol Rhinol Laryngol 2006;115:553-8.

12) Byl FM. Seventy-six cases of presumed sudden hearing loss occurring in 1973: prognosis and incidence. Laryngoscope 1977;87:817-25.

13) Chon KM, Roh HJ. The presumptive factors concerning to the prognosis of sudden deafness. Korean J Otolaryngol-Head Neck Surg 1988;31:250-8.

14) Byl FM Jr. Sudden hearing loss: eight years' experience and suggested prognostic table. Laryngoscope 1984;94:647-61.

15) Lee JH, Kim JH, Lee KP, Hong SW, Chae SY, Suh BD. Clinical analysis of sudden hearing loss. Korean J Otolaryngol 1990;33:690-7.

16) Wilson WR. The relationship of the herpesvirus family to sudden hearing loss: a prospective clinical study and literature review. Laryngoscope 1986;96:870-7.

17) Park MK, Lee JD, Lee BD, Lim HW, Hwang SJ, Chae SW. Clinical usefulness of viral IgM antibody test in sudden sensorineural hearing loss patient. Korean J Otorhinolaryngol-Head Neck Surg 2011; 54:37-42.

18) Danino J, Joachims HZ, Eliachar I, Podoshin L, Ben-David Y, Fradis M. Tinnitus as a prognostic factor in sudden deafness. Am J Otolaryngol 1984;5:394-6.

19) Ben-David J, Luntz M, Magamsa I, Fradis M, Sabo E, Podoshin L. Tinnitus as a prognostic sign in idiopathic sudden sensorineural hearing loss. Int Tinnitus J 2001;7:62-4.

20) Seo JH, Jeon EJ, Park YS, Kim J, Chang KH, Yeo SW. Meteorological conditions related to the onset of idiopathic sudden sensorineural hearing loss. Yonsei Med J 2014;55:1678-82.

21) Herbert I, Nolte E, Eichhorn T. [Weather status and incidence of idiopathic facial nerve paralyses, vestibular disorders, Menière's attacks and sudden deafness]. Laryngol Rhinol Otol (Stuttg) 1987;66: 249-50.

22) Mizukoshi K, Watanabe Y, Shojaku H, Ito M, Ishikawa M, Aso S, et al. Influence of a cold front upon the onset of Meniére's disease in Toyama, Japan. Acta Otolaryngol Suppl 1995;520 Pt 2:412-4. 